Article

\title{
The Correlation of Pore Size and Bioactivity of Spray-Pyrolyzed Mesoporous Bioactive Glasses
}

\author{
Yu-Jen Chou ${ }^{1}$, Bo-Jiang Hong ${ }^{2}$, Ying-Chih Lin ${ }^{3}$, Chen-Ying Wang ${ }^{4,5, *}$ and Shao-Ju Shih ${ }^{2, *}$ \\ 1 Department of Materials, University of Oxford, Oxford OX1 3PH, UK; yu-jen.chou@materials.ox.ac.uk \\ 2 Department of Materials Science and Engineering, National Taiwan University of Science and Technology, \\ Taipei 10607, Taiwan; M10404315@mail.ntust.edu.tw \\ 3 Horien Biochemical Technology Co., Ltd., Taichung 40768, Taiwan; esilin@biohorien.com \\ 4 School of Dentistry, National Taiwan University, Taipei 10048, Taiwan \\ 5 Department of Dentistry, National Taiwan University Hospital, Taipei 10048, Taiwan \\ * Correspondence: jywang8919@ntu.edu.tw (C.-Y.W.); shao-ju.shih@mail.ntust.edu.tw (S.-J.S.); \\ Tel.: +886-2-2312-3456 (C.-Y.W.); +886-2-2730-3716 (S.-J.S.); \\ Fax: +886-2-2383-1346 (C.-Y.W.); +886-2-2737-6544 (S.-J.S.)
}

Academic Editor: James Z. Tang

Received: 7 March 2017; Accepted: 28 April 2017; Published: 3 May 2017

\begin{abstract}
SiO}_{2}-\mathrm{CaO}-\mathrm{P}_{2} \mathrm{O}_{5}$-based mesoporous bioactive glasses (MBGs) were synthesized by spray pyrolysis in this study. Three commonly used non-ionic tri-block copolymers (L121, P123, and F127) with various lengths of hydrophilic chains were applied as structural templates to achieve different pore sizes. A mesoporous structure was observed in each as-prepared specimen, and the results showed that the L121-treated MBG had the largest pore size. The results of bioactivity tests indicated that the growth of hydroxyapatite is related to the pore size of the materials.
\end{abstract}

Keywords: mesoporous bioactive glasses; surfactants; pore size; bioactivity

\section{Introduction}

Bioactive glasses (BGs) have received considerable attention since they were developed [1]. Due to their superior bioactivity and other properties, such as non-toxicity, biocompatibility, and degradability, they have been applied to numerous tissue engineering applications, such as bone replacement, tooth repair, and drug-carrying materials [2,3]. Osteomyelitis, a serious problem, may be induced by bone filling and bone replacement during surgery [4]. A local drug delivery system providing antibiotics or growth factors needs to be introduced [5]. Therefore, a material must be bioactive and have a higher specific surface area and various pore sizes for this purpose.

In order to increase the surface area of BGs effectively, the idea of adding surfactant as a structural template to prepare mesoporous bioactive glasses (MBGs) was introduced by Yan et al. in 2004 [6]. His work successfully demonstrated the synthesis of MBGs using non-ionic tri-block copolymer based on two monomers, ethylene oxide and propylene oxide (denoted as EO and PO), which results in a well-ordered mesoporous structure and a high specific surface area of up to $300 \mathrm{~m}^{2} / \mathrm{g}$. With increased surface area, MBGs exhibit an increase in the hydroxyapatite (HA) formation rate during bioactivity tests, in which the specimens are immersed in simulated body fluid (SBF) [7]. Furthermore, the mesoporous structure has been found to be suitable for hosting and delivering various medicines. The feasibility of this concept was demonstrated by Vallet-Regi et al. in 2001 [8]. They used MBGs to host and deliver the drug ibuprofen, an analgesic drug used for treating pain, fever, and inflammation, in in vitro tests. Moreover, Perez et al. recently used MBG as the carriers to deliver growth factors and stem cells and found that the MBG carriers showed substantial improvements in cellular spreading and population up to 7 days, which provides the possible applications for tissue engineering [9]. Since 
then, many investigations have been conducted in this area to develop different types of MBGs with various porous structures and functionalities for sustained drug release.

Presently, the development of MBGs for drug delivery systems can be divided into two major parts. Numerous researchers have focused on increasing the surface area of MBGs by either varying the chemical compositions or applying different surfactants $[10,11]$. Both approaches have achieved great success in increasing the specific surface area. On the other hand, the relation between pore size and drug release rate has also been studied. Horcajada et al. demonstrated that the delivery rate decreases from 24 to $5 \mathrm{mg} / \mathrm{h}$ as the pore size decreases from 3.6 to $2.5 \mathrm{~nm}$ [12], while Vallet-Regi et al. tabulated several mesoporous materials and their drug loading abilities in 2007 [13]. However, rare studies have reported on the correlation between pore size and bioactivity, which we believe is a crucial question when it comes to applications for bone implants. For example, for tissue engineering, both drug releasing effects (pore size) [12] and bone regeneration effects (bioactivity) (Perez, et al. [9]) are important, which needs to be considered. Compared to commonly used drug-carrying materials such as MCM-41 or SBA-15, bioactive MBGs have the potential to be applied as drug-carrying materials in bone implant surgeries. Therefore, the correlation of pore size and bioactivity must be examined in relation to both the drug carrying ability and the bioactivity of MBGs.

Several studies have applied various surfactants to prepare MBGs, and numerous results have been reported [6,14-20]. However, most of the studies used sol-gel to synthesize the MBGs [11,21], and those studies were all focused on the effect of increasing the surface area on bioactivity [11,22]. In contrast, this study employed spray pyrolysis (SP), which is a continuous process with rapid heating and cooling [23]. This process preserves more metastable siloxane groups in the resulting MBGs [24]. These groups act as nucleation sites and help HA formation [25].

In this study, three MBG powders were synthesized using SP with three surfactants with different $\mathrm{EO}$ and $\mathrm{PO}$ ratios. The characterizations of phase composition, surface morphology, inner structure, and specific surface area were carried out by X-ray diffraction (XRD), scanning electron microscopy (SEM), transmission electron microscopy (TEM), and analysis of nitrogen adsorption/desorption isotherms, respectively. In addition, in vitro bioactivity was assessed by immersing the powders in SBF and evaluating the HA formation against the as-prepared glasses. Finally, the correlation between pore size and bioactivity was investigated, and pore formation mechanisms of the surfactant are proposed.

\section{Results}

\subsection{Phase Composition}

Figure 1 shows three XRD patterns of as-prepared powders treated with the L121, P123, and F127 surfactants. All XRD patterns present the absence of any crystalline phase, with only a broad band existing between $20.0^{\circ}$ and $37.0^{\circ}$. Thus, amorphous MBG powders were prepared successfully.

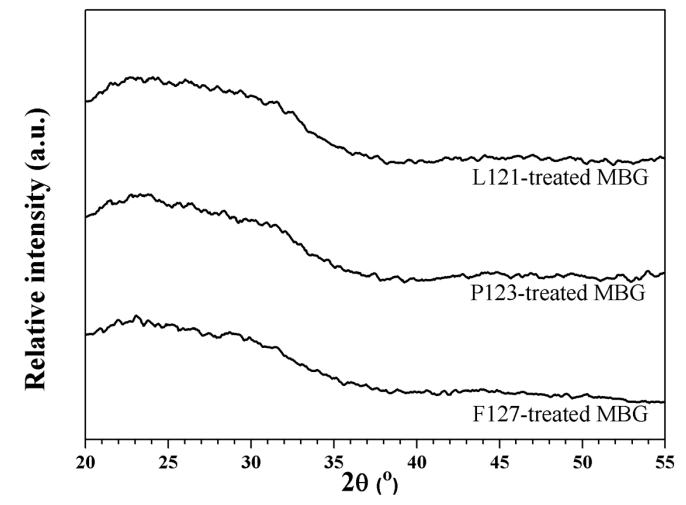

Figure 1. XRD patterns of mesoporous bioactive glass (MBG) powders treated by L121, P123, and F127 surfactants. 


\subsection{Morphology}

SEM images of L121-, P123- and F127-treated MBG powders are shown in Figure 2, demonstrating that the particles of all MBG powders were spherical. Figure 2a shows that the MBG powder treated with L121 surfactants exhibited the two morphologies of smooth sphere (denoted Type I) and wrinkled sphere (denoted Type II). The corresponding sizes of Type I and Type II particles ranged from 0.2 to $2.4 \mu \mathrm{m}$ and from 0.2 to $2.1 \mu \mathrm{m}$, respectively. For the P123- and F127-treated MBG powders, the same surface morphologies of Type I and Type II and similar particle sizes were observed, as shown in Figure 2b,c. The morphology and particle size results suggest that the three surfactant-treated MBG powders may have had the same particle formation mechanism. Furthermore, the proportions of both morphologies for all the MBG powders were measured statistically and are plotted in Figure 3. That figure shows that the volume proportions of smooth particles (Type I) were $62.5 \%, 64.9 \%$, and $81.6 \%$ for L121-, P123-, and F127-treated MBG powders, whereas the volume proportions of wrinkled particles (Type II) were $37.5 \%$, $35.1 \%$, and $18.4 \%$. The results indicated that the proportion of Type I increased (and the proportion of Type II decreased) as the EO number increased (5 for L121, 20 for P123, and 106 for F127).
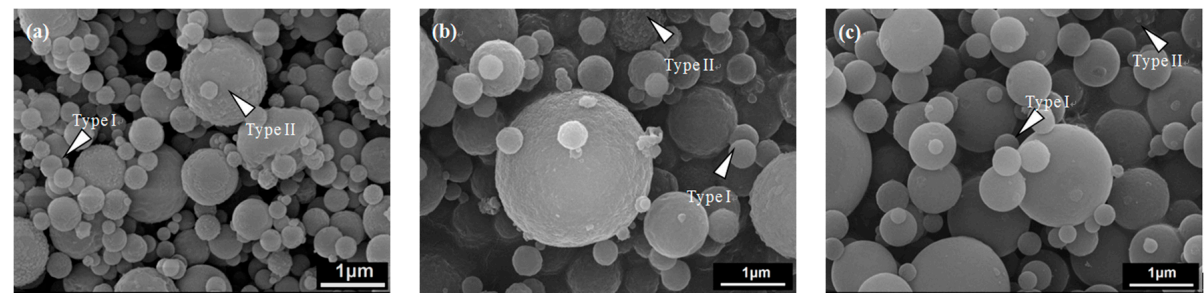

Figure 2. SEM images of (a) L121-; (b) P123-; and (c) F127- treated MBG powders.

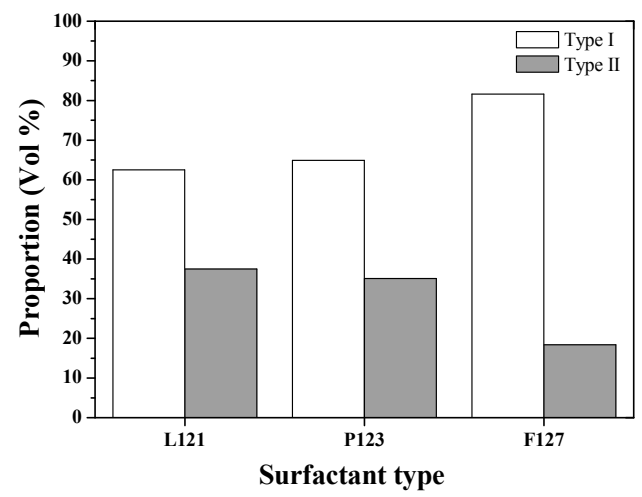

Figure 3. Particle shape volume proportion of various surfactant-treated MBG powders.

Figure 4 presents typical TEM micrographs of various surfactant-treated MBG powders. That figure confirms the morphologies of the smooth sphere and the wrinkled sphere observed by SEM (Figure 2). Furthermore, in order to observe the detailed pore structure, the illumination of the micrographs was adjusted so that they were slightly under focused, as shown in the high resolution TEM (HRTEM) images inserted in the top right corner of Figure 4. It should be noted that the contrasts in the TEM micrographs are associated with the thickness/mass of the particles. The dark contrast represents a thicker/heavier region, indicating a solid region, whereas a bright contrast represents a thinner/lighter region, indicating a pore region. Therefore, the HRTEM images in Figure 4 suggest that all MBG powders exhibited a mesoporous structure, and that pore size varied from 1 to $10 \mathrm{~nm}$. Moreover, from the HRTEM images in Figure 4, it appears that the L121-treated MBG powder had larger bright areas (i.e., larger pores) than did the P123- and F127-treated MBG powders (Figure 4b,c). This difference implies that the L121 surfactant produced the largest pores. 

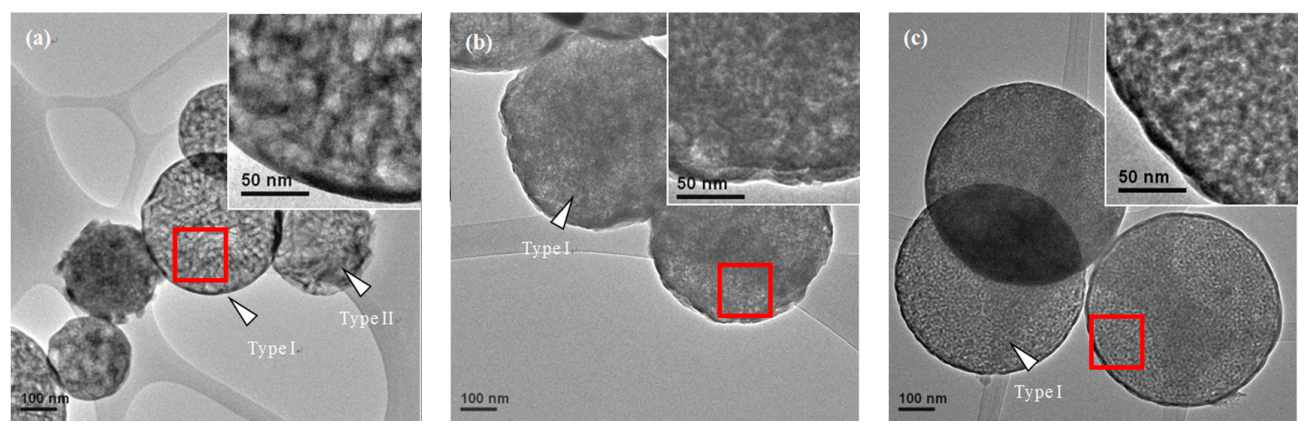

Figure 4. TEM micrographs of (a) L121-; (b) P123-; and (c) F127- treated MBG powders. Enlarged images of the MBG particles in $(\mathrm{a}-\mathrm{c})$ are shown as insets in the corresponding images.

\subsection{Particle Size Distribution and Specific Surface Area}

The pore size histograms of the surfactant-treated MBG powders are shown in Figure 5. The graphs indicate that all MBG powders exhibited a normal distribution. The pore sizes of L121-, P123-, and F127-treated MBGs ranged from 4-16, 1-10, and 1-8 nm with a decreasing trend ( $d_{50}=8.7 \mathrm{~nm}, d_{50}=4.0 \mathrm{~nm}$, and $d_{50}=4.1 \mathrm{~nm}$ for the L121-, P123- and F127-treated MBG powders). In addition, the L121-treated MBG powder demonstrated the largest pore size, $8.9 \pm 1.4 \mathrm{~nm}$, whereas the P123- and F127-treated MBG powders had smaller pore sizes of $4.5 \pm 1.0$ and $4.1 \pm 1.1 \mathrm{~nm}$, respectively. The statistical results revealed that a higher EO number of the surfactants led to a smaller pore size. Furthermore, the BET data show the pore sizes of $8.4 \pm 0.2,7.2 \pm 0.2$, and $7.2 \pm 0.2 \mathrm{~nm}$, respectively, which reveals a similar trend (L121-treated MBG $>$ P123-treated MBG $\sim$ F127-treated MBG) as the HRTEM data. Additionally, BET measurement revealed that the specific surface areas of the L121-, P123-, and F127-treated MBG powders were 137, 151, and $157 \mathrm{~m}^{2} / \mathrm{g}$, respectively, and the pore volume values for the L121-, P123-, and F127-treated MBG powders were $0.082,0.092$, and $0.079 \mathrm{~cm}^{3} / \mathrm{g}$, respectively, indicating that these three MBG powders had similar specific surface areas and pore volume values.
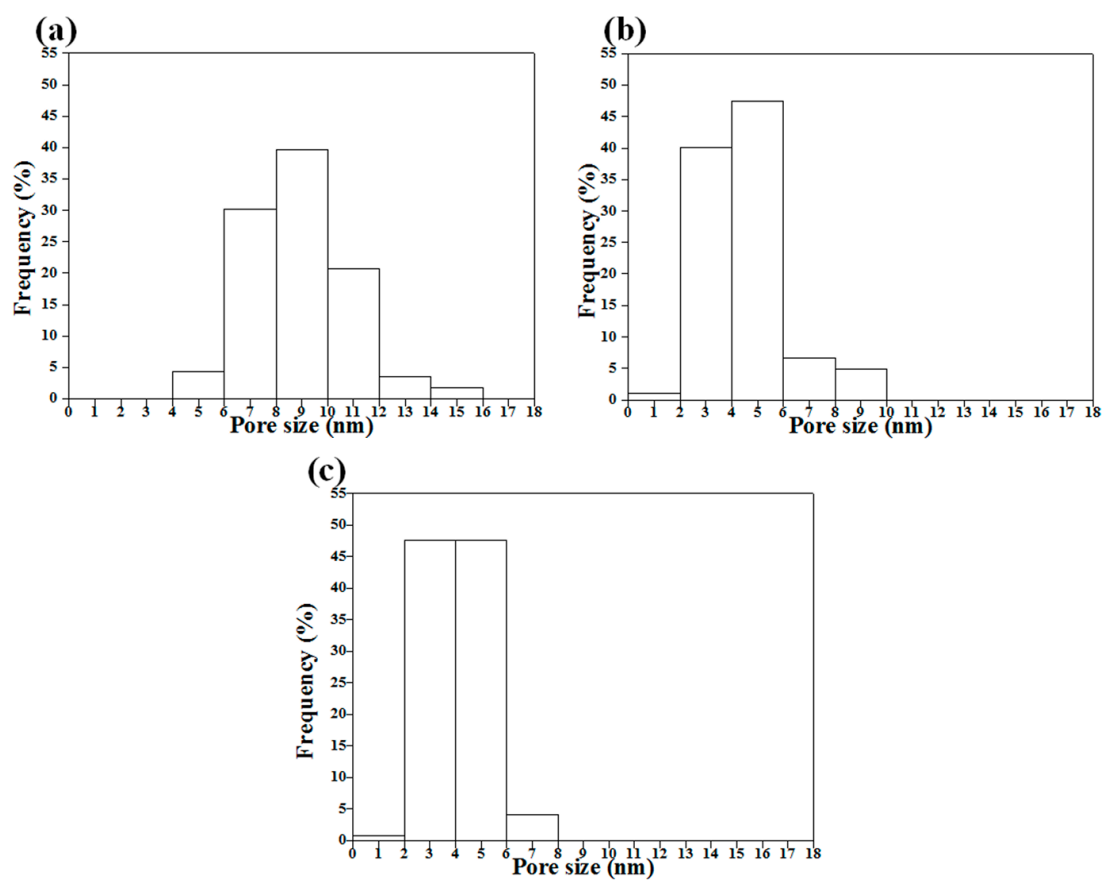

Figure 5. Pore size distributions of (a) L121-; (b) P123-; and (c) F127-treated MBG powders obtained from HRTEM images. 


\subsection{In Vitro BioactivyTests}

Bioactivity tests were carried out by XRD with all the MBG powders after immersion in SBF. After immersion in SBF for $12 \mathrm{~h}$, the growth of HA was observed with XRD patterns, as shown in Figure 6 . The diffraction peaks of $25.9^{\circ}, 28.1^{\circ}, 29.3^{\circ}, 31.8^{\circ}, 32.2^{\circ}, 33.7^{\circ}, 39.4^{\circ}, 46.7^{\circ}$, and $49.6^{\circ}$ were identified as the (002), (102), (210), (211), (112), (302), (310), (213), and (004) planes of HA (JCPDS number: 09-0432). The XRD results demonstrated that the L121-treated MBG powder had sharper and more intense peaks at (002) and (211) than did the P123- and F127-treated MBG powders, indicating higher crystallinity of HA, which corresponds to higher bioactivity [26]. To quantify the bioactivity differences, the integration of the diffraction peak area was measured. The values for the (211) peak, main HA diffraction, were 2317, 1843, and 1822 counts for the L121-, P123-, and F127-treated MBG powders, respectively. Additionally, the values for the (002) peak, the second largest peak of HA, were 1608, 1314, and 1261 counts for the L121-, P123-, and F127-treated MBG powders, following the same trend as the (211) peak. Thus, the order of both the (211) and (002) peak areas were L121-treated MBG > P123-treated MBG > F127-treated MBG. Therefore, the order of bioactivity was L121-treated MBG > P123-treated MBG > F127-treated MBG. In conclusion, the bioactivity tests showed that the surfactants with the smaller EO numbers resulted in better bioactivity.

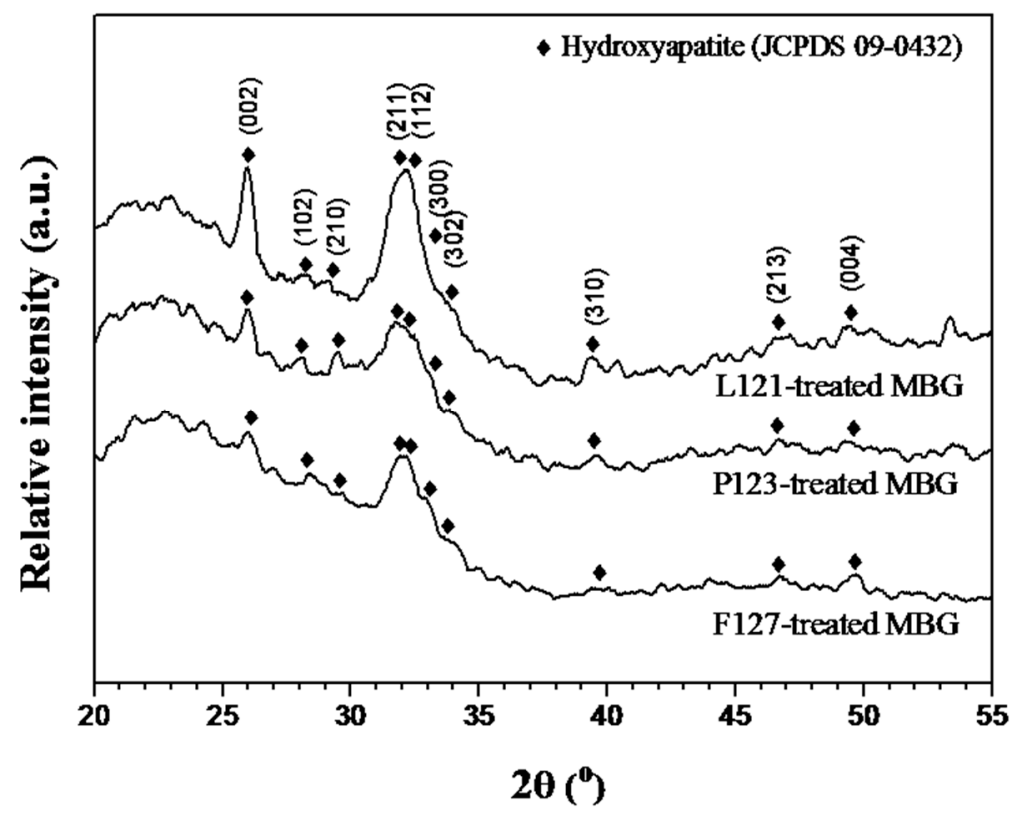

Figure 6. XRD patterns of all MBG powders after immersing in the simulated body fluid (SBF) solution for $12 \mathrm{~h}$.

Furthermore, crystallite sizes of MBG were acquired using XRD and TEM. Initially, from the XRD patterns, based on the Scherrer equation, the crystallite sizes of L121-, P123-, and F127-treated MBG powders were $22.4 \pm 12.4,16.4 \pm 1.5$, and $17.2 \pm 2.5 \mathrm{~nm}$, respectively. In addition, Figure 7 shows the TEM images and the diffraction patterns of MBG powders immersed in the SBF solution for $12 \mathrm{~h}$. To the eye, it is clear that L121-, P123-, and F127-treated MBG powders were covered by some nanocrystals; the indexed SAED patterns reveal that these nanocrystals are HA crystals. Consequently, the TEM images show that the crystallite size ranges of immersed L121-, P123-, and F127-treated MBG powders were 14-20,12-19, and 7-16 nm, which agrees with the XRD result (Figure 6). 

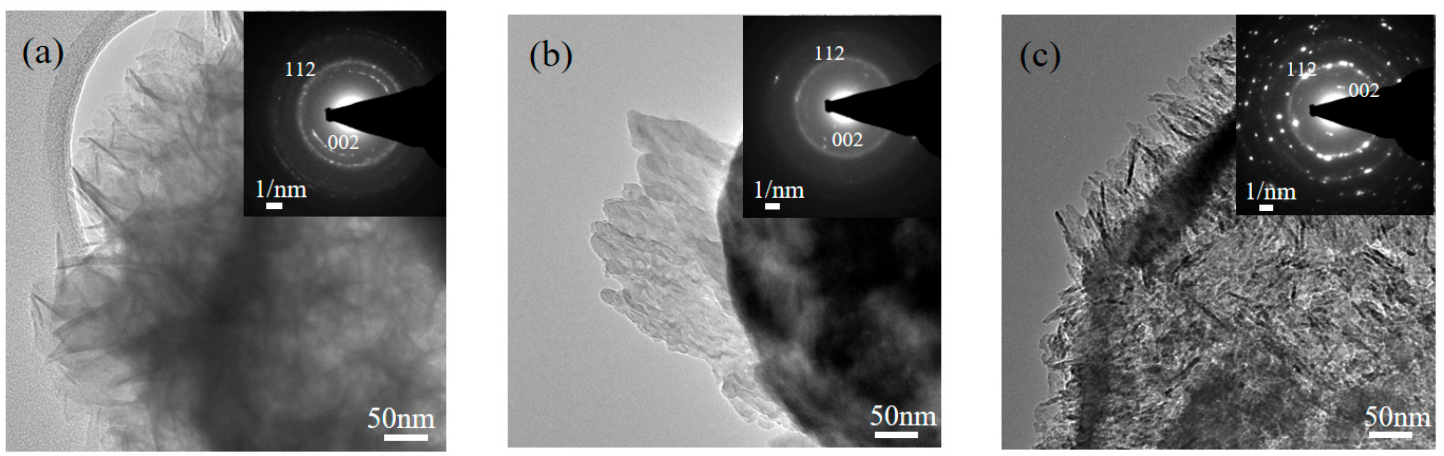

Figure 7. TEM images (a) L121-; (b) P123-; and (c) F127- treated MBG powders after immersing in SBF solution for $12 \mathrm{~h}$. The corresponding SAED pattern was inserted in the top-right corner of each figure.

\section{Discussion}

The first issue to discuss is the SP formation mechanism of MBG powders. In a typical SP process, two common mechanisms responsible for the formation of the particles are "one-particle-per-drop" and "gas-to-particle" [27]. In the former, micron-sized droplets atomized by an ultrasonic nebulizer are directly converted into oxide particles. In the latter, nano-sized particles $(<0.1 \mu \mathrm{m})$ condense from the gas phase [28]. Based on the SEM observations, all the surfactant-treated MBG powders had an average particle size of $\sim 600 \mathrm{~nm}$, indicating that all the MBG powders formed via the "one-particle-per-drop" mechanism [27]. As shown in the SEM and TEM images (Figures 2 and 4), all the MBG powders had two types of particle morphologies, the smooth sphere (Type I) and the wrinkled sphere (Type II). The Type I and Type II particles were similar in size, indicating that there was no obvious correlation between morphology and particle size. The formation of Type I particles followed the typical SP precursor mechanism of precursor volume precipitation [27,29]. The Type II particles formed because of the high surfactant concentration of those droplets, which aggregated on the surface via the SP precursor mechanism of surface precipitation [30]. After the calcination stage of the SP process, the aggregated surfactants were burned out, which resulted in the wrinkled surface morphology.

The second issue to discuss is the correlation of the EO number, the morphology, the pore size, and the bioactivity, as shown in Figure 8. To begin with, the statistical measurement of the morphology proportions, shown in Figure 3, indicated that, when the EO number of the surfactant increased, the proportion of Type II particles decreased. For example, the L121-treated MBG (EO number of 5) had the highest proportion of Type II particles, but the F127-treated MBG (EO number of 127) had the smallest proportion of these particles. As discussed above, the Type II morphology is attributed to the surfactant aggregation, and consequently, the higher proportion of Type II particles suggests more surfactant aggregation. Therefore, the L121 specimen had more aggregation than did the P123 and F127 specimens. The reason is that these three precursor solutions contained the same weight of surfactants. Since the average molar weights of L121, P123, and F127 were 4400, 5800, and 12,600, the molar concentration order of the surfactants was L121 > P123 > F127. It follows that a higher surfactant concentration increased the probability of aggregation (aggregation order of L121 > P123 > F127). Additionally, the aggregation order affected the pore size of the MBG particles. It is well known that all surfactants burn out to form pores in the calcinations step of SP, and the aggregated surfactants occupied more volume to form the larger pores. As shown in Figure 5, the pore size order of L121 > P123 > F127 followed the same trend of aggregation order, which supports this argument. Finally, a higher EO number led to a lower Type II proportion and a smaller pore size, as shown in Figure 8a. Finally, the schematic diagram of the formation mechanisms for the L121-, P123-, and F127-treated MBG powders is shown in Figure 9. 
(a)

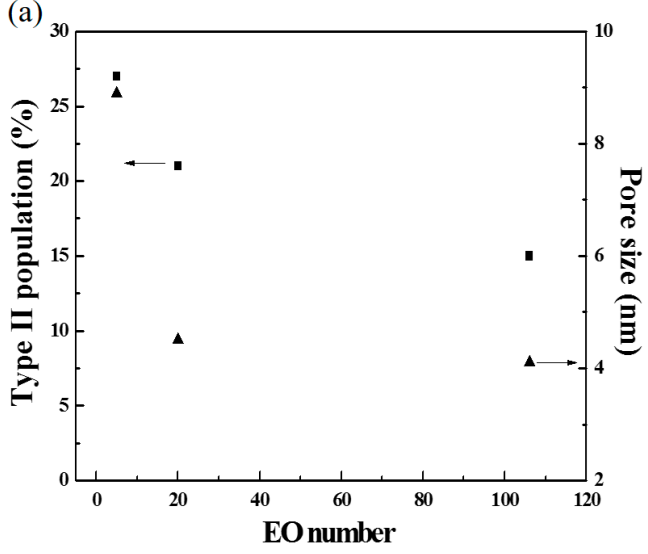

(b)

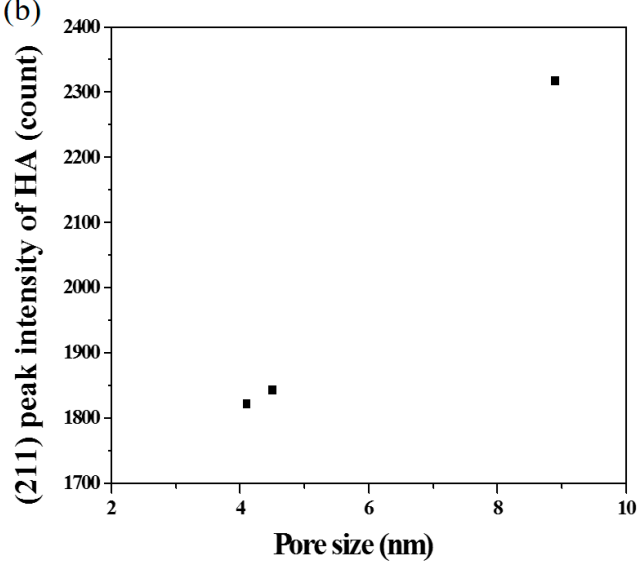

Figure 8. (a) Type II proportion and pore size as the function of EO number; and (b) the (211) peak intensity of HA as the function of pore size.

(a)

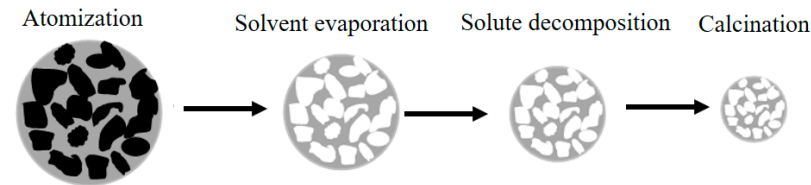

(b)

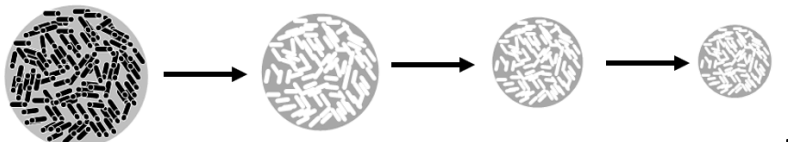

(c)

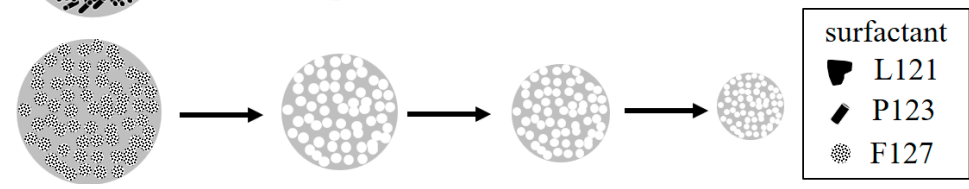

Figure 9. Schematic diagrams of formation mechanisms for the (a) L121-; (b) P123-, and (c) F127-treated MBG particle.

The third issue is bioactivity. As mentioned before, previous studies focused only on the effect of the surface area on bioactivity [11,14]. However, we believe that not only the surface area but also the pore size plays an important role in the bioactivity. Figure $8 \mathrm{~b}$ illustrates the relationship between pore size and bioactivity; it is clear that a larger pore size leads to better HA crystallinity (i.e., better bioactivity). One possible explanation is that larger pores provide more space for HA, whereas smaller pores may limit the growth of HA. In summary, MBG powders with larger pores can be prepared using surfactants with a lower EO number, and such MBG powders exhibit better bioactive properties. Therefore, they are potential candidates for drug delivery applications.

\section{Materials and Methods}

\subsection{Synthesis}

The MBG powders $\left(70 \mathrm{~mol} \% \mathrm{SiO}_{2}, 25 \mathrm{~mol} \% \mathrm{CaO}\right.$, and $5 \mathrm{~mol} \% \mathrm{P}_{2} \mathrm{O}_{5}$ ) were prepared from three distinct non-ionic tri-block surfactants of $\mathrm{L} 121\left(\mathrm{EO}_{5} \mathrm{PO}_{70} \mathrm{EO}_{5}\right.$, Sigma-Aldrich, St. Louis, MO, USA), P123 (EO ${ }_{20} \mathrm{PO}_{70} \mathrm{EO}_{20}$, Sigma-Aldrich, St. Louis, MO, USA), and F127 (EO ${ }_{106} \mathrm{PO}_{70} \mathrm{EO}_{106}$, BASF, Florham Park, NJ, USA) using SP. Tetraethyl orthosilicate (TEOS, Si( $\left(\mathrm{OC}_{2} \mathrm{H}_{5}\right)_{4}, 99.9$ wt \%, Showa, Japan), calcium nitrate tetrahydrate $\left(\mathrm{CN}, \mathrm{Ca}\left(\mathrm{NO}_{3}\right)_{2} \cdot 4 \mathrm{H}_{2} \mathrm{O}, 98.5 \mathrm{wt} \%\right.$, Showa, Japan), and triethyl phosphate (TEP, $\left(\mathrm{C}_{2} \mathrm{H}_{5}\right)_{3} \mathrm{PO}_{4}, 99.0 \mathrm{wt} \%$, Alfa Aesar, Ward Hill, MA, USA) were used as the sources for Si, $\mathrm{Ca}$, and $\mathrm{P}$, respectively. All MBG precursors, containing $44 \mathrm{wt} \%$ surfactant and $56 \mathrm{wt} \% \mathrm{Si}, \mathrm{Ca}$, and $\mathrm{P}$ precursor mixture, were dissolved in $60.00 \mathrm{~g}$ of ethanol combined with $1.00 \mathrm{~g}$ of $0.5 \mathrm{M} \mathrm{HCl}$ 
and stirred at room temperature for $24 \mathrm{~h}$ to form the final precursor solution. For the SP process, the precursor solution was dispersed into fine droplets using an ultrasonic nebulizer (King Ultrasonic Co., Taiwan) at a frequency of $1.65 \mathrm{MHz}$. The droplets went through the thermal treatments of preheating, calcining, and cooling in a tube furnace (D110, Dengyng, Taiwan) at 400,700 , and $500{ }^{\circ} \mathrm{C}$, respectively. The surfaces of the particles were then charged by electrons released from tungsten corona wire at high voltage $(16 \mathrm{kV})$. After that, the negative-charged powders were neutralized and condensed in an earthed stainless steel collector. Finally, the powders were collected and annealed at $600{ }^{\circ} \mathrm{C}$ for $1 \mathrm{~h}$ to prevent carbon contamination.

\subsection{Characterization}

Initially, the phase compositions of the MBG powders were indexed using an X-ray diffractometer (D2 Phaser, Bruker, Germany). Then, the surface morphologies and inner structures were examined with a scanning electron microscope (SEM, JSM-6500F, JEOL, Japan) and a transmission electron microscope (Tecnai G2 F20, FEI, Portland, OR, USA), respectively. In addition, the particle size distributions and pore sizes were obtained as averages of more than 100 particles and 100 pores in SEM and TEM images, respectively. The specific surface areas, pore volume, and pore sizewere measured using the Brunauer-Emmett-Teller (BET) method with nitrogen as an absorbent. The nitrogen adsorption/desorption isotherms were obtained at $-196{ }^{\circ} \mathrm{C}$ on a constant-volume adsorption apparatus (Tristar, Micromeritics, Norcross, GA, USA). As-prepared specimens were degassed at $150{ }^{\circ} \mathrm{C}$ for $3 \mathrm{~h}$ before measurements.

The in vitro bioactivity tests of the MBG powders were performed with SBF with an ionic concentration nearly equal to that of human plasma [7]. The three MBG specimens were prepared by immersing the MBG powders in SBF at a solid to liquid ratio of $1 \mathrm{mg}$ to $1 \mathrm{~mL}$ and held at $37^{\circ} \mathrm{C}$ for $12 \mathrm{~h}$, since these three specimens exhibited similar specific surface areas [31]. The powders were then washed twice with acetone and once with DI water before drying at room temperature for one day. Then, XRD, TEM, and selected area electron diffraction (SAED) were used to characterize the formation of HA.

\section{Conclusions}

Three MBG powders were prepared by SP from three distinct surfactants with various EO numbers (L121, P123, and F127), and the MBG structures, including the phase composition, morphology, pore size, and specific surface area, were characterized using XRD, SEM, TEM, and BET, respectively. The bioactive properties of these three powders were observed to be correlated to the corresponding MBG structures. The experimental data revealed that a smaller EO number of surfactant leads to (i) a higher probability of aggregation; (ii) a higher proportion of wrinkled sphere particles; (iii) a larger pore size; and (iv) better bioactivity. In summary, the relationship of MBG pore size and bioactivity has been correlated, and this correlation should provide useful information for developing future drug carriers.

Acknowledgments: The authors acknowledge the financial supports from the Ministry of Science and Technology, Republic of China (R.O.C.) (Grant Numbers MOST104-2221-E-011-017, MOST105-2221-E-011-034, and 105-2218-E-009-014).

Author Contributions: The data collection was performed by Yu-Jen Chou, Bo-Jiang Hong, and Ying-Chih Lin. The data analysis was performed by Yu-Jen Chou. The overall planning was directed by Chen-Ying Wang and Shao-Ju Shih.

Conflicts of Interest: The authors declare no conflict of interest.

\section{References}

1. Hench, L.L.; Splinter, R.J.; Allen, W.; Greenlee, T. Bonding mechanisms at the interface of ceramic prosthetic materials. J. Biomed. Mater. Res. 1971, 5, 117-141. [CrossRef]

2. Hench, L. Bioactive ceramics: Theory and clinical applications. Bioceramics 1994, 7, 3-14. 
3. Vallet-Regí, M. Revisiting ceramics for medical applications. J. Chem. Soc. Dalton Trans. 2006, 44, 5211-5220. [CrossRef] [PubMed]

4. Ciampolini, J.; Harding, K. Pathophysiology of chronic bacterial osteomyelitis. Why do antibiotics fail so often? Postgrad. Med. J. 2000, 76, 479-483. [CrossRef] [PubMed]

5. Zhu, Y.; Kaskel, S. Comparison of the in vitro bioactivity and drug release property of mesoporous bioactive glasses (MBGs) and bioactive glasses (BGs) scaffolds. Microporous Mesoporous Mater. 2009, 118, 176-182. [CrossRef]

6. Yan, X.; Yu, C.; Zhou, X.; Tang, J.; Zhao, D. Highly ordered mesoporous bioactive glasses with superior in vitro bone-forming bioactivities. Angew. Chem. Int. Ed. 2004, 43, 5980-5984. [CrossRef] [PubMed]

7. Kokubo, T.; Kushitani, H.; Sakka, S.; Kitsugi, T.; Yamamuro, T. Solutions able to reproduce in vivo surface-structure changes in bioactive glass-ceramic A-W3. J. Biomed. Mater. Res. 1990, 24, 721-734. [CrossRef] [PubMed]

8. Vallet-Regí, M. Ceramics for medical applications. J. Chem. Soc. Dalton Trans. 2001, 2, 97-108. [CrossRef]

9. Perez, R.A.; El-Figi, A.; Park, J.-H.; Kim, T.-H.; Kim, H.-W. Therapeutic bioactive microcarriers: Co-delivery of growth factors and stem cells for bone tissue engineering. Acta Biomater. 2014, 10, 520-530. [CrossRef] [PubMed]

10. Xia, W.; Chang, J. Well-ordered mesoporous bioactive glasses (MBG): A promising bioactive drug delivery system. J. Control. Release 2006, 110, 522-530. [CrossRef] [PubMed]

11. Yan, X.; Deng, H.; Huang, X.; Lu, G.; Qiao, S.; Zhao, D.; Yu, C. Mesoporous bioactive glasses. I. Synthesis and structural characterization. J. Non-Cryst. Solids 2005, 351, 3209-3217. [CrossRef]

12. Horcajada, P.; Ramila, A.; Perez-Pariente, J.; Vallet-Reg1, M. Influence of pore size of MCM-41 matrices on drug delivery rate. Microporous Mesoporous Mater. 2004, 68, 105-109. [CrossRef]

13. Vallet-Regí, M.; Balas, F.; Arcos, D. Mesoporous materials for drug delivery. Angew. Chem. Int. Ed. 2007, 46, 7548-7558. [CrossRef] [PubMed]

14. Vaid, C.; Murugavel, S.; Das, C.; Asokan, S. Mesoporous bioactive glass and glass-ceramics: Influence of the local structure on in vitro bioactivity. Microporous Mesoporous Mater. 2014, 186, 46-56. [CrossRef]

15. Yun, H.-S.; Kim, S.-E.; Hyun, Y.-T. Preparation of bioactive glass ceramic beads with hierarchical pore structure using polymer self-assembly technique. Mater. Chem. Phys. 2009, 115, 670-676. [CrossRef]

16. Zhu, Y.; Wu, C.; Ramaswamy, Y.; Kockrick, E.; Simon, P.; Kaskel, S.; Zreiqat, H. Preparation, characterization and in vitro bioactivity of mesoporous bioactive glasses (MBGs) scaffolds for bone tissue engineering. Microporous Mesoporous Mater. 2008, 112, 494-503. [CrossRef]

17. Zhao, L.; Yan, X.; Zhou, X.; Zhou, L.; Wang, H.; Tang, J.; Yu, C. Mesoporous bioactive glasses for controlled drug release. Microporous Mesoporous Mater. 2008, 109, 210-215. [CrossRef]

18. Yun, H.-S.; Kim, S.-E.; Hyun, Y.-T. Preparation of 3D cubic ordered mesoporous bioactive glasses. Solid State Sci. 2008, 10, 1083-1092. [CrossRef]

19. Yun, H.-S.; Kim, S.-H.; Lee, S.; Song, I.-H. Synthesis of high surface area mesoporous bioactive glass nanospheres. Mater. Lett. 2010, 64, 1850-1853. [CrossRef]

20. Shih, S.-J.; Chou, Y.-J.; Panjaitan, L.V.P. Synthesis and characterization of spray pyrolyzed mesoporous bioactive glass. Ceram. Int. 2013, 39, 8773-8779. [CrossRef]

21. Yan, X.; Huang, X.; Yu, C.; Deng, H.; Wang, Y.; Zhang, Z.; Qiao, S.; Lu, G.; Zhao, D. The in-vitro bioactivity of mesoporous bioactive glasses. Biomaterials 2006, 27, 3396-3403. [CrossRef] [PubMed]

22. Shih, C.-C.; Chien, C.-S.; Kung, J.-C.; Chen, J.-C.; Chang, S.-S.; Lu, P.-S.; Shih, C.-J. Effect of surfactant concentration on characteristics of mesoporous bioactive glass prepared by evaporation induced self-assembly process. Appl. Surf. Sci. 2013, 264, 105-110. [CrossRef]

23. Shih, S.-J.; Chang, L.-Y.S.; Chen, C.-Y.; Borisenko, K.B.; Cockayne, D.J. Nanoscale yttrium distribution in yttrium-doped ceria powder. J. Nanopart. Res. 2009, 11, 2145-2152. [CrossRef]

24. Shih, S.-J.; Chou, Y.-J.; Borisenko, K.B. Preparation method: Structure-bioactivity correlation in mesoporous bioactive glass. J. Nanopart. Res. 2013, 15, 1763. [CrossRef]

25. Li, R.; Clark, A.; Hench, L. An investigation of bioactive glass powders by sol-gel processing. J. Appl. Biomater. 1991, 2, 231-239. [CrossRef] [PubMed]

26. Chou, Y.-J.; Lin, S.-H.; Shih, C.-J.; Chang, S.L.; Shih, S.-J. The effect of Ag dopants on the bioactivity and antibacterial properties of one-step synthesized Ag-containing mesoporous bioactive glasses. J. Nanosci. Nanotechnol. 2016, 16, 10001-10007. [CrossRef] 
27. Messing, G.L.; Zhang, S.C.; Jayanthi, G.V. Ceramic powder synthesis by spray pyrolysis. J. Am. Ceram. Soc. 1993, 76, 2707-2726. [CrossRef]

28. Shih, S.-J.; Tzeng, W.-L.; Chou, Y.-J.; Chen, C.-Y.; Chen, Y.-J. The influence of phase separation on bioactivity of spray pyrolyzed bioactive glass. J. Nanosci. Nanotechnol. 2015, 15, 4688-4696. [CrossRef] [PubMed]

29. Liou, S.-H.; Tsou, T.-C.; Wang, S.-L.; Li, L.-A.; Chiang, H.-C.; Li, W.-F.; Lin, P.-P.; Lai, C.-H.; Lee, H.-L.; Lin, M.-H. Epidemiological study of health hazards among workers handling engineered nanomaterials. J. Nanopart. Res. 2012, 14, 878. [CrossRef]

30. Chen, X.; Mao, S.S. Titanium dioxide nanomaterials: Synthesis, properties, modifications, and applications. Chem. Rev. 2007, 107, 2891-2959. [CrossRef] [PubMed]

31. Shih, S.-J.; Lin, Y.-C.; Valentino Posma Panjaitan, L.; Rahayu Meyla Sari, D. The correlation of surfactant concentrations on the properties of mesoporous bioactive glass. Materials 2016, 9, 58. [CrossRef]

(C) 2017 by the authors. Licensee MDPI, Basel, Switzerland. This article is an open access article distributed under the terms and conditions of the Creative Commons Attribution (CC BY) license (http:/ / creativecommons.org/licenses/by/4.0/). 